\title{
Chiral properties of domain wall fermions with improved gauge actions
}

\author{
Konstantinos Orginos ${ }^{\text {a }}\left[\mathrm{RBC}\right.$ Collaboration ${ }^{*}$

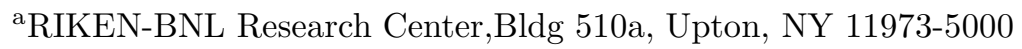

\begin{abstract}
We study the chiral properties of quenched domain wall fermions with several gauge actions. We demonstrate that the nearly translationally invariant modes in the fifth dimension that dominate the residual mass for Wilson gauge action can be substantially suppressed using improved gauge actions. In particular, we study the Symanzik action, the Iwasaki action, the DBW2 action and compare them to the Wilson action.
\end{abstract}

\section{INTRODUCTION}

Domain wall fermions provide a very elegant solution to the problem of chiral fermions on the lattice; in the limit of infinite length $L_{s}$ of the fifth dimension chiral symmetry becomes exact even when the lattice spacing does not vanish. Since practical implementations can only deal with finite $L_{s}$ some residual chiral symmetry breaking is induced. This breaking goes to zero as $L_{s}$ goes to infinity. It is therefore important to chose the parameters of the simulation so that an acceptably small chiral symmetry breaking is achieved at a reasonably small $L_{s}$.

Recently, RBC [1] and CP-PACS [2,3] reported that by using the Iwasaki gauge action, rather than the Wilson action, a significant reduction of the residual chiral symmetry breaking is achieved for the same $L_{s}$ and lattice spacing $a$. It is instructive therefore to study the effects of the gauge action in a systematic way.

We report here our results from our studies of the residual chiral symmetry breaking for the 1loop Symanzik, Iwasaki [4], and DBW2 actions. The DBW2 action was introduced in [5] and it was shown by QCD-TARO in [6] that it is a good approximation of the RG flow on the two dimensional plane of the plaquette and rectangle couplings.

All the above gauge actions can in general be

\footnotetext{
${ }^{*}$ We thank RIKEN, Brookhaven National Laboratory and the U.S. Department of Energy for providing the facilities essential for the completion of this work.
}

written as

$$
\begin{aligned}
S_{G}= & \frac{\beta}{3}\left(c_{0} \sum_{x ; \mu<\nu} P_{\mu \nu}+c_{1} \sum_{x ; \mu \neq \nu} R_{\mu \nu}+\right. \\
& \left.+c_{2} \sum_{x ; \mu<\nu<\sigma} C_{\mu \nu \sigma}\right)
\end{aligned}
$$

where $P_{\mu \nu}$ is the standard plaquette in the $\mu, \nu$ plane, and $R_{\mu \nu}$ and $C_{\mu \nu \sigma}$ denote the real part of the trace of the ordered product of SU(3) link matrices along $1 \times 2$ rectangles and $1 \times 1 \times 1$ paths, respectively. For the 1-loop Symanzik action the coefficients $c_{0}, c_{1}$, and $c_{2}$ are computed in tadpole improved one loop perturbation theory [7]. While $c_{2}=0$ and $c_{0}=1-8 c_{1}$ for both Iwasaki and DBW2, $c_{1}=-0.331$ and -1.4067 respectively.

As a measure of the chiral symmetry breaking we use the so called residual mass. The residual mass is defined as

$m_{\mathrm{res}}=\left.\frac{\left\langle J_{q}^{5}(0) J^{5}(t)\right\rangle}{\left\langle J^{5}(0) J^{5}(t)\right\rangle}\right|_{t \geq t_{\text {min }}}$,

where $J_{q}^{5}$ is the mid-point chiral symmetry breaking term which appears in the axial Ward identity of domain wall fermions, and $t_{\min }$ is sufficiently large to avoid short-distance lattice artifacts.

In addition, for each configuration we have defined the ratio

$R(t)=\frac{J_{q}^{5}(0) J^{5}(t)}{J^{5}(0) J^{5}(t)}$

which is very useful in monitoring the chiral symmetry breaking on each configuration. 
Table 1

The simulation parameters for all the actions

\begin{tabular}{lllll}
\hline Action & $\beta$ & \multicolumn{1}{c}{$M_{\rho}$} & $M_{5}$ & \multicolumn{1}{c}{$L_{s}$} \\
\hline Wilson [8] & 6.00 & $0.404(8)$ & 1.8 & $12-24$ \\
Symanzik & 8.40 & $0.411(14)$ & 1.8 & 16 \\
Iwasaki & 2.60 & $0.415(13)$ & 1.8 & 16 \\
DBW2 & 1.04 & $0.399(11)$ & 1.7 & $8-16$ \\
\hline
\end{tabular}

\section{MEASUREMENTS - RESULTS}

We measured the light hadron spectrum, the residual mass, and the ratio $R(t)$ of Eq. 3 on $16^{3} \times 32$ lattices. We used 50 Symanzik and Iwasaki lattices, and 90 DBW2 lattices. For all the actions tested the lattice spacing was tuned to $a^{-1} \sim 2 \mathrm{GeV}$ using the $\rho$ mass at the chiral limit to set the scale. This matches the Wilson gauge action at $\beta=6.0$. The corresponding $\beta$ values are given in Table 1. We have optimized the value of the domain wall height $M_{5}$. For all actions except the DBW2, the optimum value of $M_{5}$ was found to be 1.8. For the DBW2 action the optimum $M_{5}$ is 1.7 , which is what we used for the results reported below.

In Figure 1 we plot the the ratio of eq. 3 summed over time slices 4 to 16 . As we can see, the Wilson and Symanzik action data (squares and stars) show very large fluctuations. But the Iwasaki and DBW2 show a baseline of small fluctuations interrupted by spikes. Presumably the large fluctuations observed for the Wilson and Symanzik action are due to a very high frequency of spikes. The important feature of Figure 1 is that the number of spikes is drastically reduced for the DBW2 action. It is expected that these spikes are spatially localized and occur on configurations that have eigenvalues of the transfer matrix in the 5 th dimension very close to 1 . We have confirmed these expectations by measuring both the spacial structure of the spikes and studying the spectral flow of the 4D Wilson Dirac operator on these configurations (see talk by T. Izubuchi at this conference).

In Fig. 2 we show the $L_{s}$ dependence of the residual mass. All data are for $a^{-1}=2 \mathrm{GeV}$ and bare quark mass $m_{q}=0.02$. The value of $\frac{M_{\pi}}{M_{\rho}}$ is

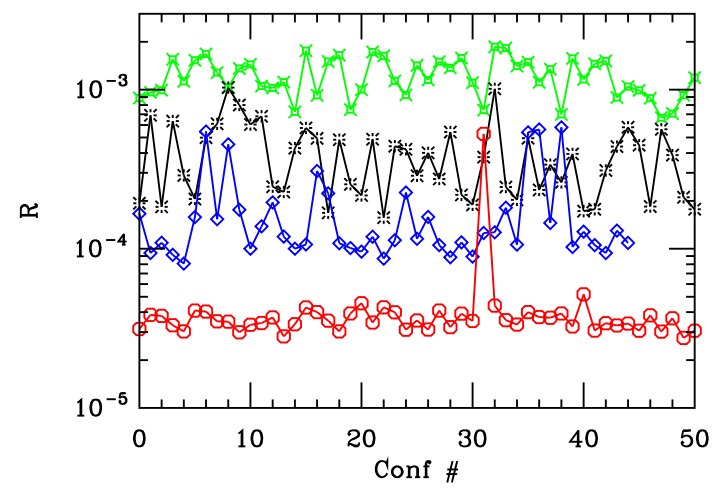

Figure 1. $R(t)$ for bare quark mass 0.02 summed over t from 4 to 16 . The fancy squares correspond to Wilson action, the bursts to Symanzik, the diamonds to Iwasaki and the octagons to DBW2.

within $10 \%$ of .55 . Since we are interested in differences by factors of ten, and since we know that the $m_{\text {res }}$ depends mildly on the quark mass [8.3], the data can be directly compared as is. For completeness we also present the CP-PACS residual mass results [3]. We have confirmed their results for $L_{s}=16$ (diamond in Fig. 2). For the Symanzik action we ran at $L_{s}=16$ only; the residual mass turns out to be smaller by a factor of three than the Wilson residual mass at the same $L_{s}$, and it is larger by a factor of two than that of the Iwasaki action. The DBW2 action not only gives the smallest residual mass, but also has the steepest decrease as a function of $L_{s}$. Since we have only three points, we cannot claim that we know the asymptotic behavior; it is interesting to note however, that a simple fit gives us $m_{\text {res }}(s) \sim q^{s}$ with $q \sim 0.6$. Shamir's one loop perturbative [9] result is $q=0.5$.

For the DBW2 action, in addition to the residual mass tests we have also measured the light hadron spectrum and pseudoscalar decay constants at both $2 \mathrm{GeV}$ and $1.3 \mathrm{GeV}$. The residual mass at $1.3 \mathrm{GeV}$ is about a factor of two smaller than the Wilson residual mass at $2 \mathrm{GeV}$ $\left(L_{s}=16\right)$. This allows simulations at coarser lattice spacing and at moderate values of $L_{s}$ with 


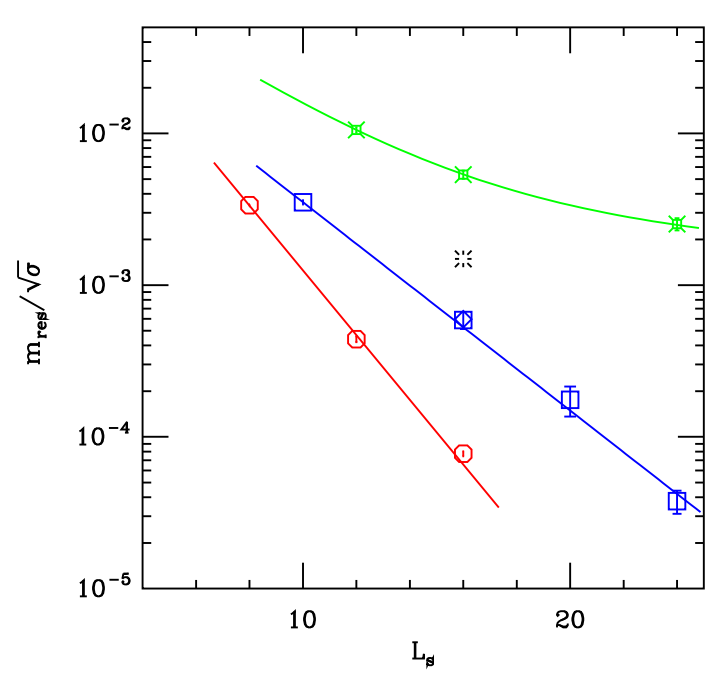

Figure 2. $L_{s}$ dependence of $m_{\text {res }}$ at $a^{-1} \sim 2 \mathrm{GeV}$. The octagons correspond to the DBW2 action, the squares (CP-PACS [3]) and diamond (RBC) to Iwasaki, the burst to Symanzik, and the fancy squares to Wilson.

minimal chiral symmetry breaking. Our spectrum results show that the already very good scaling of domain wall fermions with Wilson gauge action is preserved with the DBW2 action. For a detailed presentation of our spectrum data see the talk by Y. Aoki at this conference.

It has been argued 10] that the enhancement of chiral symmetry breaking is caused by configurations which also represent changing topology (i.e. small instantons and/or dislocations). Our data (Fig. 1) indicate that both the Iwasaki and the DBW2 actions suppress these configurations. In fact, Iwasaki [- 4 has argued that his choice of the $c_{1}$ coefficient is on the boundary separating the space of actions which have stable instantons and those which have unstable instantons. Consequently, when these improved actions are used, the question whether the topology changes arises. We have measured the topological charge on all the actions we tested, and we have concluded that the topology changes with a significantly lower rate for the DBW2 action. The problem gets more severe as we get closer to the continuum limit. The Iwasaki action also shows signs of slow topology changing. We believe that this slowdown in the topology change can be overcome by using over-relaxed heatbath algorithm and by simply running for a long time before saving configurations. Given the considerable cost of measuring fermionic observables with DWF we can afford to do so.

\section{CONCLUSIONS}

Using the DBW2 action, we have essentially eliminated the problem of residual chiral symmetry breaking for domain wall fermions at $2 \mathrm{GeV}$ with $L_{s}=16$. The DBW2 residual mass is about an order of magnitude smaller than the Iwasaki $m_{\text {res }}$, and two orders of magnitude smaller than the Wilson $m_{\text {res }}$. Assuming a $440 \mathrm{MeV}$ string tension, the bare DBW2 residual mass is about $30 \mathrm{KeV}$. The observed topology change slowdown may be handled by using longer running between measurements. Finally, our results suggest that the DBW2 action should also be useful for overlap fermions, since it probably makes the approximation to the sign function converge faster by eliminating the small eigenvalues of the Hermitian Wilson Dirac operator.

\section{REFERENCES}

1. L.-l. Wu, Nucl. Phys. Proc. Suppl. 83, 224 (2000).

2. A. Ali Khan et al., Nucl. Phys. Proc. Suppl. 83, 591 (2000).

3. A. Ali Khan et al., Phys. Rev. D63, 114504 (2001).

4. Y. Iwasaki, unpublished UTHEP-118 (1983).

5. T. Takaishi, Phys. Rev. D54, 1050 (1996).

6. P. de Forcrand et al., Nucl. Phys. B577, 263 (2000).

7. M. G. Alford et al., Phys. Lett. B361, 87 (1995).

8. T. Blum et al., hep-lat/0007038 (2000).

9. Y. Shamir, Phys. Rev. D62, 054513 (2000).

10. R. Narayanan, Nucl. Phys. Proc. Suppl. 73, 86 (1999). 